\title{
A Rare Case Report of Eumycetoma Osteomyelitis of Hand
}

\author{
Dr. Ann Rose Thomas ${ }^{1 *}$, Dr. Jayalakshmi PL ${ }^{2}$, Dr. Sankar $S^{3}$
}

${ }^{1}$ Junior Resident Pathology, ${ }^{2}$ Associate Professor Pathology, ${ }^{3}$ Professor and HOD Pathology, Govt. Medical College Kottayam, Kerala, India

\author{
DOI: $\underline{10.36348 / \mathrm{sipm} .2021 . v 06 i 02.008}$ \\ | Received: 08.02.2021 | Accepted: 22.02.2021 | Published: 28.02.2021 \\ *Corresponding author: Dr. Ann Rose Thomas
}

\section{Abstract}

Mycetoma is a chronic, suppurative, granulomatous infection of subcutaneous tissue that eventually may involve bone. The Infection is caused by free living, exogenous, geophilic actinomycetes and fungi. It is characterized by a triad of localized swelling, underlying sinus tract and production of grains or granules. Approximately $40 \%$ of mycetomas worldwide are eumycotic and rest is actinomycotic [1]. Eumycetoma is most probably acquired by traumatic inoculation of certain fungi into subcutaneous tissue. Fungi are considered as an unusual cause of osteomyelitis of hand/palm. This paper describes a case of eumycetoma osteomyelitis of the hand. With clinical, radiological and pathological correlation we arrived at a final diagnosis.

Key words: Osteomyelitis, eumycetoma, hand, immunocompromised.

Copyright (C) 2021 The Author(s): This is an open-access article distributed under the terms of the Creative Commons Attribution 4.0 International License (CC BY-NC 4.0) which permits unrestricted use, distribution, and reproduction in any medium for non-commercial use provided the original author and source are credited.

\section{INTRODUCTION}

Eumycetoma is a chronic progressively destructive morbid inflammatory disease caused by various genera of fungi. This disease is characterized by marked progressive destruction of soft tissue and bone causing functional disability. This is not a contagious disease and all the victims are infected from sources in nature.

Once the offending agent begins to develop in tissue,its mycelium proliferates and becomes organized into aggregates called granules/grains. These granules are the hallmark of mycetoma. Granules are of different size, colours and degrees of hardness depending on the species causing mycetoma. As the mycetoma develops, the affected area enlarges as a result of the interaction of the host and the organism and the formation of fibrotic tissue. Abscesses develop and multiple draining sinuses eventually emerge. Discharge from sinuses contains grains composed of mycelial filaments (2-4 micron), broad hyphae and bizarre forms and chlamydospores.

\section{CASE REPORT}

A 64 year old Carpenter, Who was a case of diabetes mellitus on oral hypoglycemic agents, complained of difficulty in folding left hand for the past 4 years. Then he developed an initially painless swelling over dorsum of (L) hand, which eventually developed into a painful abscess with multiple draining sinuses with purulent discharge. The patient had no systemic manifestations or other skeletal complaints. Blood and serum examinations were also unremarkable.

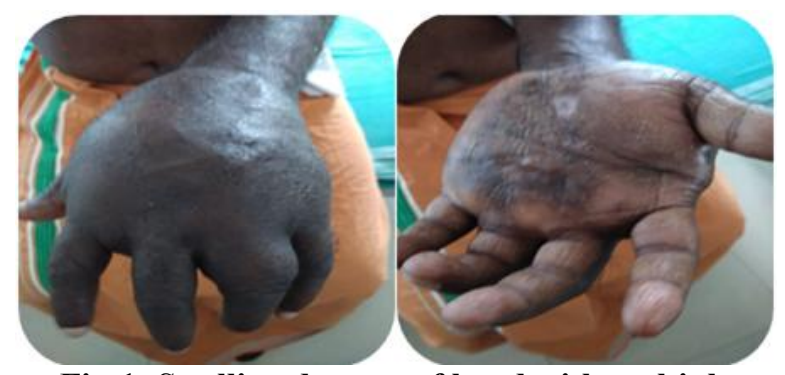

Fig-1: Swelling dorsum of hand with multiple sinuses

Radiological and pathological examination

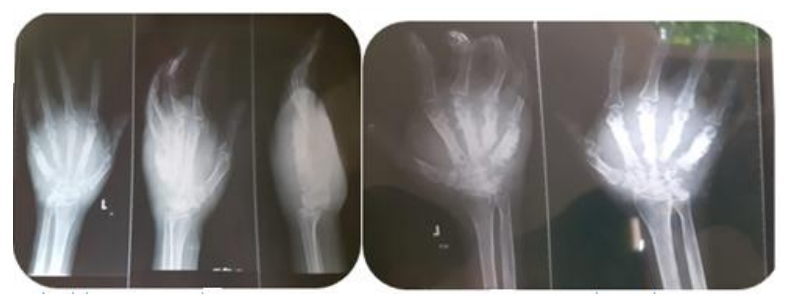

Fig-2: Massive bony destruction and soft tissue swelling

X-ray finding of hand revealed massive bony destruction of the carpel and metacarpal bones of (L) hand consistent with chronic osteomyelitis. 
Histopathological examination showed multiple bony trabeculae with marrow spaces showing dense chronic inflammatory infiltrates and focal collection of neutrophils and at its center, radiating filaments surrounded by dense eosinophilic material deposition(Splendore hoeppli phenomenon) seen. No granuloma or no evidence of malignancy noted.

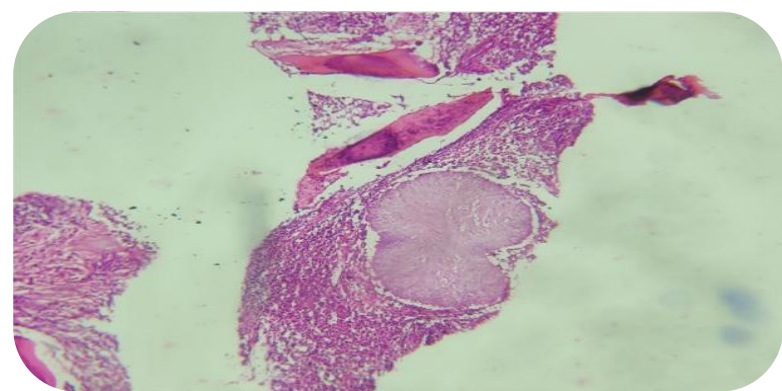

Fig-3: H\&E Splendore Hoeppli Phenomenon

PAS and GMS stain showed acute angled branched hyphae with septations. Gram stain - Positive and Modified ZN stain was negative. A diagnosis of Eumycetoma osteomyelitis was made basded on morphology.

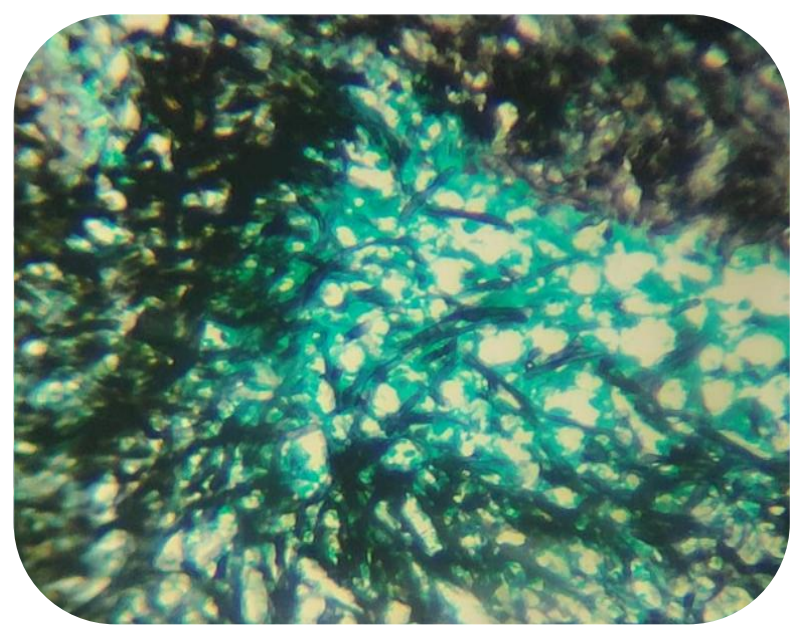

Fig-4: GMS (Positive)

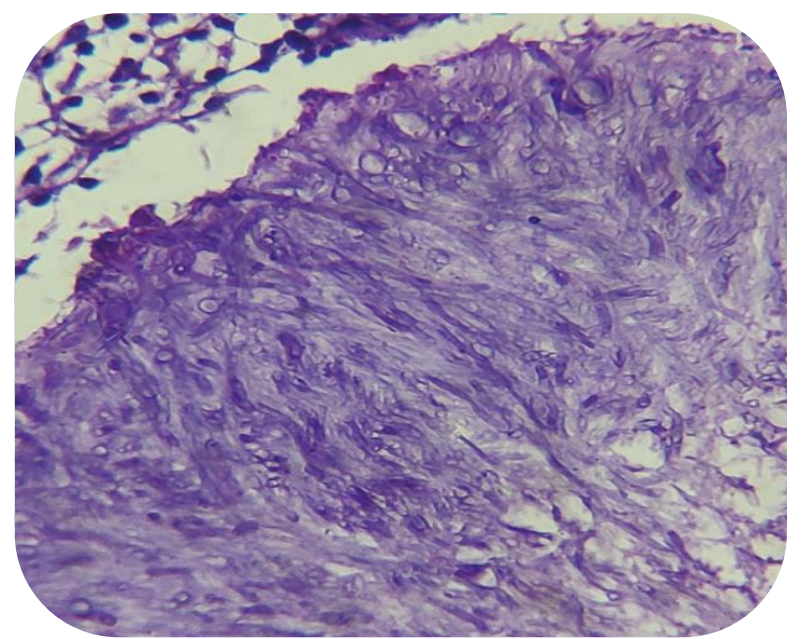

Fig-5: PAS stain (positive)

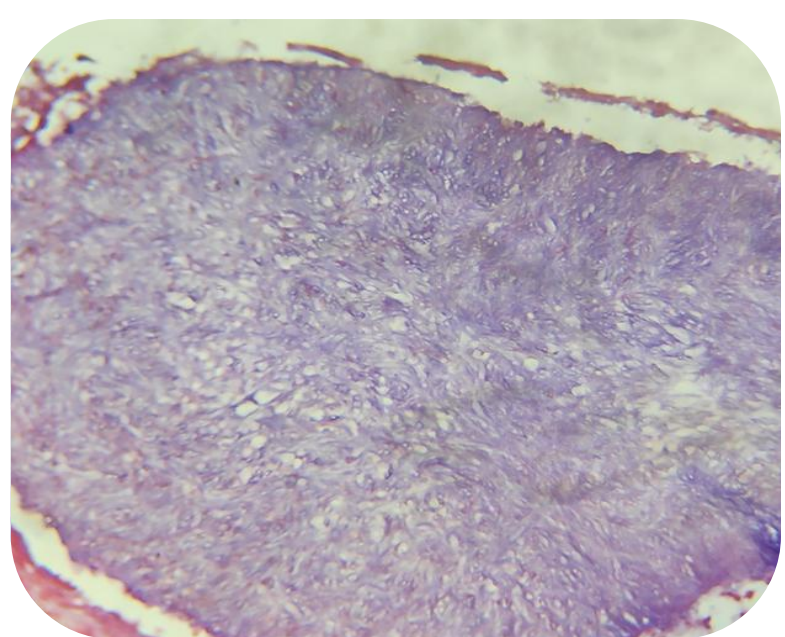

Fig-6: Gram stain (positive)

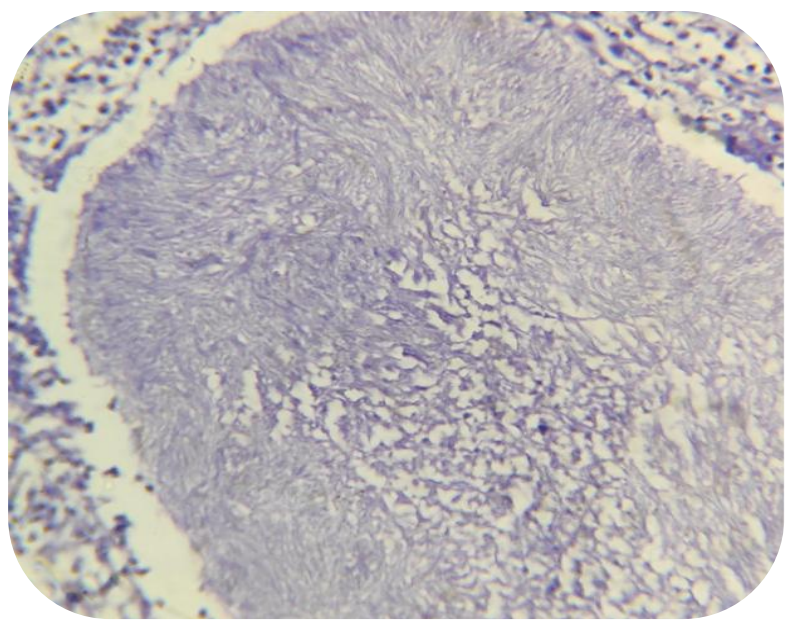

Fig-5: Modified Ziehl Neelsen stain

Microbial Culture: No fungal pathogen isolated from culture (Patient was on antibiotics and antifungals)

\section{DISCUSSION}

Eumycetoma osteomyelitis of hand is very rare and very difficult to differentiate it from other bacterial osteomyelitis clinically.

Agents that cause eumycetoma are primarily saprophytic microorganisms that are found in soil and on plant matter. Healthy persons become inoculated with these agents as a result of the traumatic implantation of the thorns, splinters and other plant matter. The incidence of mycetoma is more particularly occupation dependent and mainly a disease of the tropical and sub-tropical zones. Madurella mycetomatis accounts for most cases Worldwide.

Eumycetoma may present as a small localized swelling with or without sinuses or can be associated with significant morbidity in terms of gradual enlargement, destruction and deformity of the affected 
site. They are prone to infect immunocompromised individuals.

The eumycetoma can be differentiated from actinomycetoma when discharging grains are visible to the naked eye. The grains vary in colour, size and consistency depending on the causative agent. Granules of eumycetoma are firm 0.2-5 mm aggrigate of organized vegetative, septate hyphae, which often are embedded in a matrix cement substance of eumycetoma, producing black granules. Actinomycotic granules are composed of mycelial filaments of $<1$ micron. Actinomycetes are Gram stain positive, Giemsa positive, GMS positive and PAS negative, but eumycetes are GMS and PAS positive.

The study of discharged granules crushed on the slide and stained with special stains, allows differentiation between thin filaments of actinomycetoma and thicker hyphae of eumycetoma.

Fungi stains well with GMS and PAS may or may not take up giemsa and gram stain. Confirmation of diagnosis and species identification of organism can be done by culture studies [2]. Imaging studies will show osteolytic activity (bone destruction) in both cases.

Actinomycotic mycetoma responds well to medical management (Antibiotics). Eumycotic mycetoma needs long term systemic antifungals that may not be completely effective in all cases and requires surgical management (combined approach of both medical and surgical therapy).
Radiological examination is essential for followup of patients with bone involvement $\&$ on medical treatment in order to assure cure.

\section{CONCLUSION}

An appropriate diagnosis of eumycotic mycetoma requires a combination of clinical, radiological pathological and microbiological studies [3]. As the chemotherapeutic options for actinomycetoma and eumycetoma vary, the clinician must confirm the diagnosis before starting the treatment [4]. This case report highlights the fact that morphological diagnosis of eumycetoma is possible with the help of histochemical stains.

\section{REFERERENCES}

1. El-Sobky, T. A., Haleem, J. F., \& Samir, S. (2015). Eumycetoma osteomyelitis of the calcaneus in a child: a radiologic-pathologic correlation following total calcanectomy. Case reports in pathology, 2015.

2. Afroz, N., Khan, N., Siddiqui, F. A., \& Rizvi, M. (2010). Eumycetoma versus actinomycetoma: Diagnosis on cytology. Journal of Cytology/Indian Academy of Cytologists, 27(4), 133.

3. Yadav, T., Meena, V. K., Shaikh, M., Khera, S., Sureka, B., Garg, P., \& Khera, P. S. (2020). Clinico-radiological-pathological correlation in eumycetoma spectrum: Case series. Northern Clinics of İstanbul, 7(4), 400.

4. Relhan, V., Mahajan, K., Agarwal, P., Garg, V.K. (2017). Mycetoma: An update. Indian Journal of Dermatology, 1;62(4):332. 